\title{
Reducing Muscle Soreness and Muscle Damage: A Role for Branched-Chain Amino Acids
}

\author{
Amber Petchonka1 ${ }^{*}$, Bill I Campbell ${ }^{1}$, and Jennifer A Bunn ${ }^{2}$ \\ ${ }^{1}$ School of Physical Education and Exercise Science, University of South Florida, Tampa, Florida, USA \\ ${ }^{2}$ Department of Exercise Science, Campbell University, Buies Creek, North Carolina, USA
}

Unaccustomed or highly intense exercise can result in muscle damage. Muscle damage is associated with morphological changes, increases in serum Creatine Kinase (CK) concentrations, increases in myoglobin, decreases in subsequent exercise performance, and Delayed Onset Muscle Soreness (DOMS). In particular, eccentric exercise causes myofibrillar disruption and primarily damages the fast twitch fibers, although research is beginning to show it can affect slow twitch fibers as well [1]. Due to the damage that follows unaccustomed, intense, or eccentrically biased exercise, a focus on recovery strategies that mitigate these unwanted effects should be pursued. Recovery from a bout of exercise can emphasize many variables, including:

- Replenishing skeletal muscle glycogen

- Rehydration

- Reducing muscle soreness

- Attenuating serum markers of muscle damage

Recovery from training has been categorized into 3 domains: immediate recovery, short-term recovery, and training recovery [2]. Training recovery is the recovery between two individual training sessions or competitions, and is best utilized for prevention of or elimination of DOMS. The majority of the studies looked at throughout this article have training sessions ranging from several days to several weeks.

DOMS occurs 24-48 hours after exercise and is a type I muscle strain which results in a dull pain and muscle stiffness. DOMS peaks 24-72 hours after the unaccustomed exercise and is most often measured using serum Creatine Kinase (CK) levels, myoglobin levels, muscle tenderness, or a Visual Analogue Scale (VAS). Cleary et al. assessed differences in muscle soreness from two eccentric exercise bouts that were completed 6-9 weeks apart [3]. The study targeted the wrist extensor muscles of the subject's non-writing forearm where participants performed 5 sets of maximal eccentric repetitions of the wrist extensor muscles with a 60 -second rest period between sets. DOMS was assessed using VAS scores and muscle tenderness via a punctuate tenderness gauge. The results showed a lower VAS score and lower muscle tenderness within 24 hours after the second bout of eccentric exercise when compared to the first. The authors concluded that a prior exercise stimulus may decrease DOMS with subsequent exercises. Similarly, Jamurtas et al. assessed if there were differences in DOMS between plyometric, concentric, and eccentric exercises [4]. DOMS was assessed subjectively with VAS and by measuring serum CK levels. Subjects in the concentric and eccentric groups performed six sets of leg extensions and six sets of calf raises at 70\% of 1rep-max, and completed repetitions to volitional exhaustion. The plyometric group performed six sets of drop jumps and six sets of side jumps over a rope, where box and rope height were set at $70 \%$ of their 1rep-max jump height. Results from this study revealed that subjective muscle soreness was higher for the plyometric and eccentric exercise groups than the concentric group. However, there was no difference in serum CK levels between the groups. Together, these two studies revealed that DOMS is most likely induced through eccentric and plyometric exercise. Additionally, both studies showed an increase in subjective ratings of DOMS and muscle tenderness with these exercises, although there was no change in serum CK levels.
Branched Chain Amino Acids (BCAA's) include leucine, isoleucine and valine, which are essential amino acids that need to be consumed through diet or supplementation because they are not produced in the body. BCAA consumption is often associated with muscle protein synthesis and promoting an anabolic setting in the body after exercise. Because of this anabolic effect, consuming BCAA's may help alleviate muscle soreness and suppress muscle damage. A study by Jackman et al. addressed the effects of BCAA consumption on muscle soreness caused by eccentric exercise [5]. Participants either consumed the BCAA supplement (3.5 $\mathrm{g}$ of Leucine, $2.1 \mathrm{~g}$ Isoleucine, $1.7 \mathrm{~g}$ Valine) or a placebo 30 minutes prior to exercise, 1.5 hours after exercise, two more times on the exercise day, and four times on the following two days. Results showed no difference in muscle soreness between groups, but plasma CK concentrations and myoglobin increased after exercise and remained elevated through the entire exercise protocol for both groups. The authors concluded that despite the anabolic effects of BCAA supplementation, it did not attenuate blood markers for muscle soreness with eccentric-exercise induced DOMS.

Shimomura et al. examined the effects of BCAA supplementation on squat exercise and DOMS [6]. Supplementation included: $5.5 \mathrm{~g}$ of a BCAA, $1 \mathrm{~g}$ of instant green-tea powder and $1.2 \mathrm{~g}$ of artificial sweetener containing aspartame dissolved in $200 \mathrm{ml}$ of de-ionized distilled water, which was consumed the morning of the testing. VAS showed muscle soreness for both groups on days two and three of the study, but the ratings for the BCAA group were significantly lower than those of the placebo group. A significant change in myoglobin concentrations was observed in the placebo trial and not the BCAA trial, suggesting that muscle damage was less in the BCAA trial. Similarly, Sharp and Pearson looked at the effects of overreaching while participants consumed NutriBuild II capsules consisting of: $2000 \mathrm{mg}$ glutamine, $1800 \mathrm{mg}$ leucine, $750 \mathrm{mg}$ isoleucine, and $750 \mathrm{mg}$ of valine; taking 6 pills in the morning and 6 pills in the evening with meals for three weeks [7]. In the fourth week, the participants completed a resistance training regimen. Serum cortisol concentrations were significantly lower for the BCAA group compared to the placebo group at two and four days of training and 36 hours after the last session of training. Total CK concentrations were also significantly lower for the BCAA group then the placebo group.

DOMS is a regular and painful part of progressing and overloading muscles in order to achieve changes in strength. In an effort to alleviate muscle soreness, research indicates that BCAA supplementation may

*Corresponding authors: Amber Petchonka, Bill I Campbell, School of Physical Education and Exercise Science, University of South Florida, Tampa, Florida, USA E-mail: Amber.Petchonka@mosaicco.com, Campbell@coedu.usf.edu

Received September 04, 2012; Accepted September 04, 2012; Published September 07, 2012

Citation: Petchonka A, Campbell BI, Bunn JA (2012) Reducing Muscle Soreness and Muscle Damage: A Role for Branched-Chain Amino Acids. J Sports Med Doping Stud 2:e125. doi:10.4172/2161-0673.1000e125

Copyright: (c) 2012 Petchonka A, et al. This is an open-access article distributed under the terms of the Creative Commons Attribution License, which permits unrestricted use, distribution, and reproduction in any medium, provided the original author and source are credited. 
Citation: Petchonka A, Campbell BI, Bunn JA (2012) Reducing Muscle Soreness and Muscle Damage: A Role for Branched-Chain Amino Acids. J Sports Med Doping Stud 2:e125. doi:10.4172/2161-0673.1000e125

be effective in decreasing muscle soreness in many types of exercises, as well as promoting an anabolic environment that is often desired with resistance training. While an exact amount for optimal BCAA supplementation is not known, it is suggested that physically active individuals do not need to consume large amounts of BCAA to reduce muscle soreness [7]. Instead, it appears that consistent ingestion of BCAA's is helpful in reducing DOMS.

\section{References}

1. Campbell BI, LaBounty PM, Wilborn CD (2011) Dietary Supplements used in combat sports. Strength and Conditioning Journal 33: 50-59.

2. Howatson G, van Someren KA (2008) The prevention and treatment of exercise-induced muscle damage. Sports Med 38: 483-503.
3. Cleary MA, Kimura IF, Sitler MR, Kendrick ZV (2002) Temporal pattern of the repeated bout effect of eccentric exercise on delayed-onset muscle soreness. J Athl Train 37: 32-36.

4. Jamurtas A, Fatouror I, Buckenmeyer P (2000) Effect of plyometric exercise on muscle soreness and plasma creatine kinase levels and its comparison with eccentric and concentric exercise. J Strength Cond Res 14: 68-74.

5. Jackman S, Witard O, Jeukendrup A, Tipton KD (2010) Branched-chain amino acid ingestion can ameliorate soreness from eccentric exercise. Med Sci Sports exerc 42: 962-970.

6. Shimomura $Y$, Inaguma A, Watanabe S, Yamamoto $Y$, Muramatsu $Y$, et al (2010) Branched-chain amino acid supplementation before squat exercise and delayed-onset muscle soreness. Int J Sport Nutr Exerc Metab 20: 236-244.

7. Sharp CP, Pearson DR (2010) Amino acid supplements and recovery from high-intensity resistance training. J Strength Cond Res 24: 1125-1130. 